\title{
A descrição do funcionamento de um motor Homopolar linear e suas aplicações: Ilustrando o funcionamento de um acelerador de partículas
}

The description of the operation of a linear homopolar motor and applications: Illustrating the operation of a linear particle accelerator

\author{
Adriano Doff*1, Romeu M. Szmoski ${ }^{1}$ \\ ${ }^{1}$ Departamento Acadêmico de Física, Universidade Tecnológica Federal do Paraná, Ponta Grossa, PR, Brasil
}

Recebido em 15 de dezembro de 2015. Revisado em 6 de janeiro de 2016. Aceito em 17 de janeiro de 2016

Neste trabalho exploramos o potencial didático de um motor homopolar linear com o objetivo de ilustrar o princípio de funcionamento de um acelerador de partículas. A fim de estabelecer o mecanismo de funcionamento de um motor homopolar linear, consideramos uma analogia com a descrição de um motor homopolar rotatório, e partir deste apresentamos os princípios de funcionamento deste dispositivo e estabelecemos um limite teórico para a velocidade do motor linear. De posse dos dados gerados experimentalmente para a velocidade do elemento móvel do motor, cujo o resultado está em conformidade com o limite teórico estabelecido, ilustramos o princípio de funcionamento de um acelerador de partículas linear (LINAC). Palavras-chave: Imãs de neodímio, motor elétrico linear, tracker, acelerador de partículas.

In this work we explored the didatic potential of a linear homopolar motor for the purpose of illustrating the principle of operation of a particle accelerator. In order to establish the operating mechanism of a linear electric motor, we consider an analogy with the description of a rotary homopolar motor and from this we present the operating principles of this device and we establish a theoretical limit on the speed of the linear motor. Assuming experimental results for the speed of the movable element of the system, whose result is in accordance with the theoretical limit, we illustrate the operating principle of a linear particle accelerator (LINAC).

Keywords: Neodymium magnets, linear electric motor, tracker, particle accelerator.

\section{Introdução}

Motores são dispositivos que convertem alguma forma de energia em energia mecânica. Especificamente os motores elétricos operam de modo a transformar a energia elétrica em energia cinética rotacional ou translacional. Motores elétricos cujo resultado é a energia cinética rotacional são denominados rotativos e são os mais convencionais. Por outro lado, se a conversão resulta em energia mecânica sob a forma de movimento de translação o motor é dito linear. Diante disso classificamos o dispositivo proposto neste trabalho como motor linear homopolar. O conceito homopolar é motivado pela

*Endereço de correspondência: agomes@utfpr.edu.br descrição apresentada na Ref. [1] e maiores detalhes sobre este motor será apresentado na seção seguinte.

Popularmente conhecido na internet como "trem elétrico" ou "trem eletromagnético", o motor homopolar linear é um dispositivo composto por imãs de neodimio grudados nas extremidades de uma pilha e um conjunto de espiras construídas com fios de cobre desencapado que servem de trilho para o objeto se deslocar. Vários vídeos demonstrando este dispositivo em movimento podem ser facilmente encontrados na internet [2]. No entanto, ainda há pouco ou quase nenhum trabalho que explore de maneira teórica e didática os mecanismos físicos envolvidos. Versões consideradas mais simples foram minuciosamente investigadas por diferentes pesquisadores [1, 3, 4] e, recentemente Stephen Irons [5], apre- 
sentou um trabalho específico sobre funcionamento deste motor, no entanto, embora seja específico sobre o motor linear sua descrição é ainda de cunho qualitativa.

Neste trabalho propomos explorar o potencial didático do motor linear homopolar e descrever sua dinâmica subjacente. Para isso tomaremos como base, principalmente, a Ref. [1] considerando o motor linear como um análogo translacional de um motor elétrico homopolar rotacional. Esta analogia, nos permitará delinear uma descrição detalhada da dinâmica de operação do motor bem como obter uma equação para a velocidade do carrinho móvel. Para testar nossa proposta e previsão teórica determinaremos também a velocidade a partir da análise e tratamento dos vídeos do dispositivo usando o programa Tracker [6]. Por último, como uma aplicação da potencialidade deste dispositivo, ilustramos o princípio de funcionamento de um acelerador de partículas , que consiste de um tema mais avançado e que pode ser utilizado como um exemplo da aplicação de conhecimentos elementares a descrição de sistemas físicos mais complexos.

Devido a proposta didática do trabalho este foi dividido da seguinte forma: Na seção 2 apresentamos os conceitos básicos destinados a elaboração de um motor homopolar linear. Na seção 3 apresentamos os princípios físicos necessários para a descrição do movimento do conjunto móvel do motor linear e, empregando uma analogia com o movimento do motor homopolar, elaboramos uma descrição detalhada da dinâmica de operação do motor linear. Na seção 4 apresentamos os resultados experimentais obtidos para a descrição do conjunto móvel do motor linear proposto. Por fim, na seção 5, com base na dinâmica do motor linear, ilustramos o princípio de funcionamento de um acelerador de partículas linear (LINAC), a seção 6 contêm nossas conclusões.

\section{Elaboração de um motor elétrico ho- mopolar linear}

Como mencionamos anteriormente os motores elétricos podem, com base no tipo de movimento que produzem, ser classificados em rotativos e lineares. Além disso, um motor linear pode ser visto como um motor rotativo tradicional que teve o seu estator (parte estacionária do motor rotativo) cortado radialmente e depois desenrolado. A Fig. 1, extraida da Ref. [7, representa pictoricamente a construção/definição do motor linear que descrevemos acima.

Uma ilustração do sistema proposto é apresentada na Fig.2. Dado que o sistema deve produzir um movimento translacional trata-se de um motor linear e que, como veremos, possui um grande apelo didático. Como pode ser observado na figura, tratase de uma espira circular (solenóide) formada por

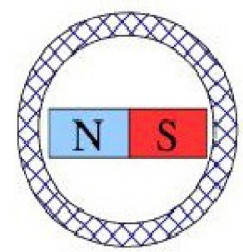

(a)

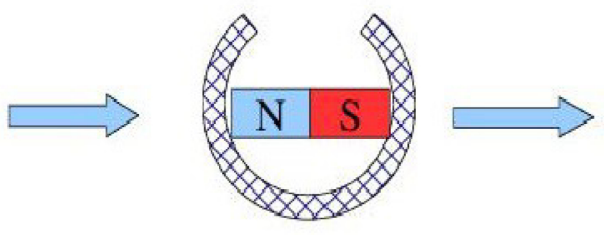

(b)

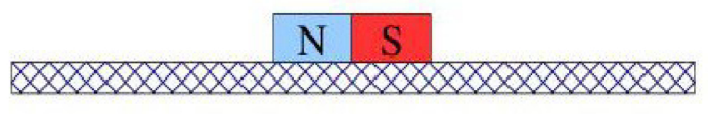

(c)

Figura 1: Representação idealizada da construção de um motor linear

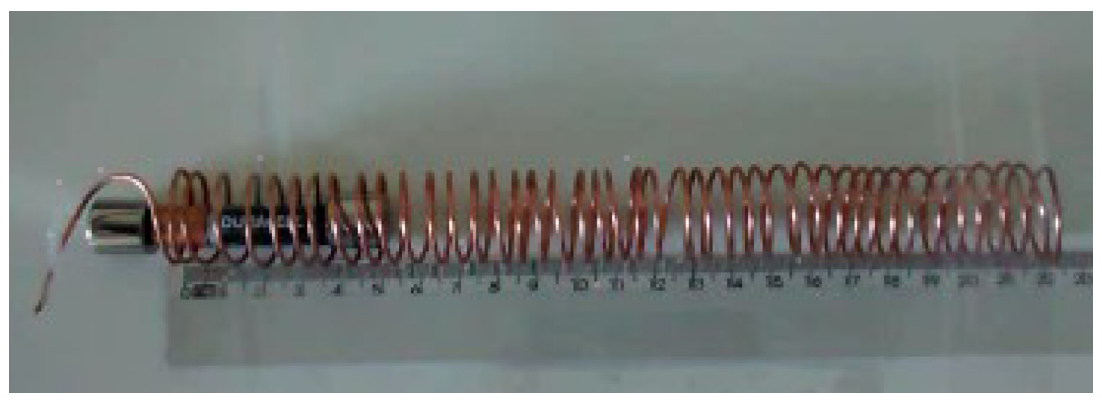

Figura 2: Motor linear didático empregado no experimento 
fio elétrico desencapado de $\mathrm{d}=2,5 \mathrm{~mm}$ de diâmetro, uma pilha alcalina AAA e dois potentes imãs de neodímio. O solenóide, como mencionamos anteriormente, faz o papel de estator e, aqui, possui comprimento $L=21,5 \mathrm{~cm}$ e diâmetro interno $D=2,0 \mathrm{~cm}$. Na Fig. 2 a pilha alcalina AAA (1,6V e $1600 \mathrm{mmA} . \mathrm{h})$ é acoplada a dois potentes imãs de neodimio, os quais são revestidos por um material condutor de forma que ao entrarem em contato com o interior do solenóide estabelecem o fechamento do circuito elétrico que corresponde a região do solenóide englobada pelo conjunto (imã + pilha). O movimento da pilha no interior do solenóide é análogo ao movimento de um motor homopolar usual (rotatório) para o movimento de translação, sendo uma consequência direta da interação do campo do imã com o campo estabelecido na região do solenóide delimitado pelo conjunto (imã + pilha)[ilha onde o comportamento de $\vec{B}_{\text {sol }}$ é não-uniforme], após ser estabelecido a corrente gerada pela pilha. Conforme comentamos na seção introdutória o motor considerado neste trabalho têm a finalidade de ilustrar o princípio fundamental de funcionamento de um acelerador de partículas, tema que será desenvolvido na seção 5. Antes disso, nas seções subsequentes, iremos descrever a dinâmica de funcionamento do motor e apresentar os resultados obtidos para o movimento descrito pelo do motor linear proposto.

\section{Princípios físicos e caracterização do movimento do motor linear}

O princípio básico em que os motores eletromagnéticos são baseados reside no fato que quando um fio condutor, imerso em uma região de campo magnético perpendicular ao eixo de percurso da corrente, experimenta uma força dada por

$$
\vec{F}=I \oint d \vec{l} \times \vec{B}
$$

onde $I$ é a corrente que atravessa o fio, $\vec{B}$ representa o campo magnético local atuante sobre o fio e $d \vec{l}$ um elemento de comprimento. Em um motor elétrico usual (rotatório) o rotor gira pois os fios e o campo magnético são arranjados de forma que ocorra um torque sobre a linha central do rotor. Conforme comentamos na seção anterior, o motor elétrico linear considerado pode ser visto como o análogo translacional de um motor elétrico homopolar usual, como o "roller"homopolar descrito na Ref. [1]. A apresentação do princípio de funcionamento de um motor homopolar está muito bem apresentada na referência indicada, em particular, o roller descrito nesta referência irá fornecer a base para a descrição da dinâmica do motor linear considerado . Um ponto fundamental para a descrição da dinâmica do motor homopolar consiste na observação que os imãs, na forma de cilindro que formam o roller, ao girar criam uma força eletromotriz

$$
|\varepsilon(\omega)|=\frac{1}{2} B r^{2} \omega
$$

devido ao fluxo magnético criado em consequência da rotação. Esta (fem) induzida, por sua vez, irá criar uma corrente contrária a corrente fornecida pela pilha a fim de conservar a energia, levando portando a

$$
I=\frac{V_{\text {pilha }}-B r^{2} \omega}{R}
$$

onde $V_{\text {pilha }} \sim 1,6 V, R$ é a resistência do circuito que representa o motor e $B$ o campo magnético criado por cada imã. De posse desta informação, chega-se, como em [1] à seguinte equação de movimento para a componente tangencial $v_{t}$ do "roller"homopolar

$$
\frac{d v_{t}(t)}{d t}=\frac{B r^{3}}{\bar{I}}\left(\frac{V_{p i l h a}-B r v_{t}}{R}\right)-\hat{a} \mu_{d y n}
$$

sendo $\bar{I}$ o momento de Inércia do sistema (imãs + pilha), $\hat{a}=\frac{4 r^{2}}{3 \bar{I}} N=100 \mathrm{~m} / \mathrm{s}^{2}$ e $\mu_{d y n}$ o coeficiente de atrito dinâmico do conjunto com a superficie em que o "roller"gira e $v=\omega r$. Na Fig. 3 apresentamos a solução numérica da Eq.(4), em função da variação do coeficiente atrito dinâmico (eixo y) e o tempo (eixo x). Esta figura reflete bem o comportamento observado para variação da componente tangencial da velocidade de rotação do "roller"em função de um intervalo assumido para o coeficiente de atrito dinâmico. Dado que, após um certo intervalo de tempo o motor entra no regime estacionário $\left(v_{t}=\right.$ constante $)$, fato este garantido pela condição $a \rightarrow 0$, um ponto que deve ser exclarecido nesta figura é que esta descreve a região física de parâmetros, representada por cores de tom azul claro a escuro no canto superior esquerdo, correspondente a $0,012<\mu_{d y n}<0,019$, uma vez que neste intervalo as curvas que representam a velocidade tangencial tendem a atingir a condição quaseestacionária para um intervalo de tempo compatível com o observado para este tipo de motor, o que não ocorre para outra região do gráfico. Para as cores 
em tom claro, $\mu_{d y n} \rightarrow 0$, o regime estacionário é apenas alcançado para $t \rightarrow \infty$, ainda com base nesta é possível estimar que a velocidade do "roller"homopolar para o intervalo de tempo $\Delta t=4 \mathrm{~s}$, que foi considerado pelo autor em [1], estaria compreendida entre $0,34 \mathrm{~m} / \mathrm{s}<v_{t}<1,2 \mathrm{~m} / \mathrm{s}$, onde o resultado experimental é $v_{t}^{e x p}=0,525 \mathrm{~m} / \mathrm{s}$ que está em muito bom acordo com a previsão teórica. Um resultado mais refinado para o intervalo de velocidade dependerá de uma determinação mais precisa do coeficiente de atrito dinâmico do sistema.

Notando que a força tangencial exercida pelo campo magnético do imã é $F(B)=I B r$, e que $\bar{I} \approx M r^{2}$ a Eq. $[4$ pode ser colocada na forma

$$
\frac{d v_{t}(t)}{d t}=\frac{F(B)}{M} I(V, \omega)-\hat{a} \mu_{d y n}
$$

A equação diferencial acima descreve de forma razoável o comportamento da velocidade terminal do "roller"homopolar. Devido a usual analogia entre o movimento de rotação e translação, podemos esperar que o motor linear considerado neste trabalho também possa ser descrito por uma equação do mesmo tipo, a saber,

$$
\frac{d v(t)}{d t}=\frac{F(B)}{M} f(I, V, v(t))-g \mu_{d y n}
$$

sendo $F(B)$ a força magnética sentida pelo conjunto (imãs + pilha) devido a interação do campo produzido pelos imãs de neodímio com o solenóide, $f(I, V, v(t))$ uma função, em princípio desconhecida, que irá depender da corrente, da ddp e da velocidade, e $\mu_{d y n}$ o coeficiente de atrito dinâmico, o qual assumimos satisfazer a equação $f_{\text {atrit }}=\mu_{d y n} M g$. A caracterização das quantidades elencadas é um problema difícil, que dependerá justamente do conhecimento detalhado de como o campo estabelecido pelos imãs de neodímio interagem com o campo gerado na "ilha"magnética estabelecida na região delimitada pelo conjunto (imãs + pilha). Conforme comentamos, este trabalho tem um apêlo didático e o motor linear considerado tem a finalidade de ilustrar o princípio de funcionamento de um acelerador de partículas linear e, portanto, iremos assumir um modelo bastante simplicado para a caracterização das funções desconhecidas. Com o objetivo de ilustrar as condições de funcionamento do motor linear na Fig. 4, apresentamos um solenóide muito grande e isotrópico, formado por (k) seções de espiras, onde inserimos na ( $j$-ésima) seção um conjunto de dois imãs que iremos assumir apresentar o mesmo comportamento (simetrico) na configuração das linhas de campo magnético, porém, separados por uma peça plástica de comprimento (l) grande o suficiente apenas para os campos gerados pelos magnetos não interagirem.

Após estabelecido a posição do conjunto de imãs no solenóide, vamos também assumir que uma ddp

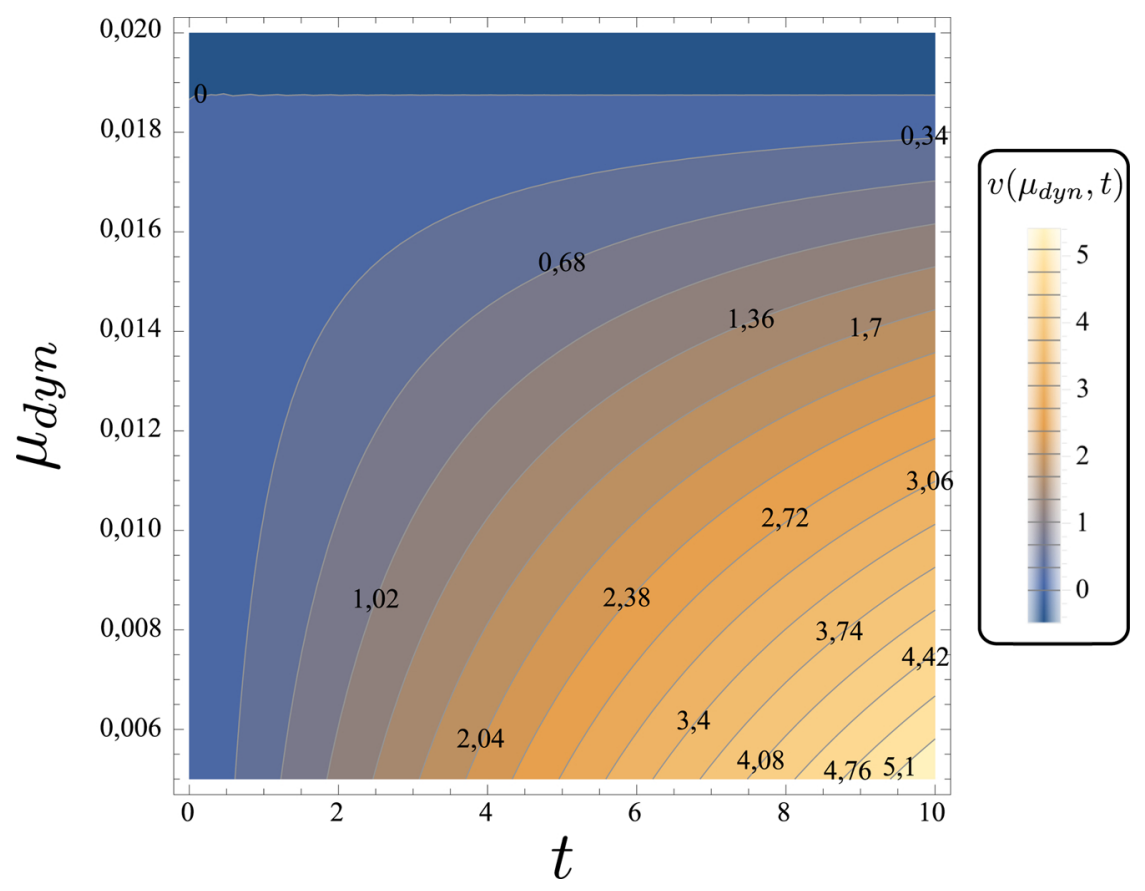

Figura 3: Comportamento de $v_{t}(t)$ em função da força de atrito dinâmico e do tempo 


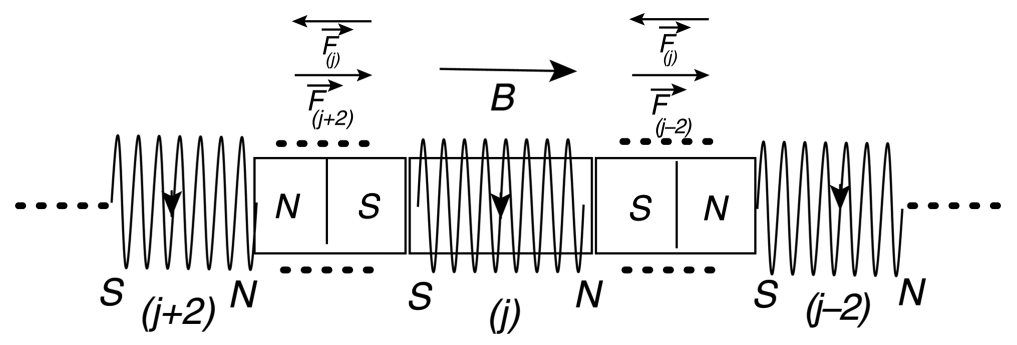

Figura 4: Ilustraçao esquemática do mecanismo de operação do motor homopolar linear

seja aplicada de forma a configurar uma corrente (I) no solenóide, cuja a orientação esta indicada na respectiva figura. O campo magnético gerado no interior do solenóide $(\vec{B})$ é orientado na direção $(+\hat{i})$ e as linhas pontilhadas (....) nos extremos representam que este se estende por longas distâncias, e as linhas pontilhadas intermediárias representam as seções $((j+1),(j-1))$ que foram retiradas apenas para proporcionar a vizualização dos magnetos. Neste caso o campo magnético estabelecido no interior do solenóide é uniforme ( ou seja é o mesmo) em qualquer seção $(j-k, j+k)$ e descrito por

$$
\vec{B}=\mu_{0} n I \hat{i}
$$

onde $n=\frac{N}{L}, N$ o número total de espiras e $\mu_{0}$ a permeabilidade magnética do vácuo. $\mathrm{O}$ imã na posição $(j+1)$ sente uma força $\left(-\hat{i} \vec{F}_{j}\right)$ devido a espira posicionada em $(j)$, a uniformidade do campo magnético no solenóide implica que a força associada a seção $(j+2)$ em módulo é igual a $\left|\vec{F}_{j}\right|$, porém, de orientação contrária $\vec{F}_{j+2}=+\hat{i} \vec{F}_{j}$ que leva a uma força resultante nula sobre o magneto $\left(\vec{F}_{j+1, R}=\vec{F}_{j}+\vec{F}_{j+2}=0\right)$. O problema é simétrico em relação a seção $(j)$, de forma que a força resultante sobre o magneto situado na seção $(j-1)$ tambêm é nula e o conjunto de magnetos fica em repouso. Entretanto, vamos considerar agora uma segunda situação, onde a peça plástica que conecta os magnetos é substituida por uma pilha AAA cuja a orientação de seus pólos leve a uma corrente (I) de mesmo sentido representado na Fig. 4, a diferença é que neste caso apenas a espira contida na seção $(j)$ apresentará um campo magnético, por isso o comentário apresentado na seção anterior a respeito da região delimitada pelo conjunto (imãs+pilha) se comportando como uma "ilha"onde o comportamento de $\vec{B}_{s o l}$ é não uniforme, neste caso podemos dizer que a simetria observada anteriormente, $\left(\vec{F}_{j+k, R}=\vec{F}_{j-k, R}=0\right)$, não será mais respeitada já que o campo no centro da seção (j) do solenóide não será o mesmo das extremidades, ou seja $\vec{B}_{c}>\vec{B}_{e}$. Em consequência desta não - uniformidade do campo magnético nas seções de espira é que movimento do motor elétrico linear têm início e se propaga na direção $(-\hat{i})$, uma outra forma de chegar a este resultado seria empregando o princípio da conservação da energia, uma vez que variação da densidade de energia magnética no meio implica na seguinte força sobre cada magneto, $\vec{F}=-\vec{\nabla} U_{m}$, para um campo uniforme $\vec{\nabla} U_{m}=0$ os magnetos permanencem em repouso, porém, para a segunda situação considerada ganhamos $\vec{\nabla} U_{m} \neq 0$ e que caracteriza o movimento dos magnetos. Como comentamos a caracterização das funções descritas pela Eq. (6) é um problema difícil, que dependerá justamente do conhecimento detalhado de como o campo estabelecido pelos imãs interage com o gerado na "ilha"magnética. Um modelo simplificado para o comportamento de $\vec{B}_{c}$ e $\vec{B}_{e}$ pode ser obtido a partir da Ref. 8. Nesta os autores descrevem o comportamento da força produzida por um solenóide sobre um núcleo de ferrite móvel que, após ser magnetizado, apresenta um campo $\vec{B}_{\text {ferr }}=k \mu_{0} \hat{i}$. Uma vez que para materiais ferromagnéticos a permeabilidade relativa $(k>>1)$, podendo estar na faixa $\left(10^{2}<k<10^{7}\right)[9$ podemos considerar que o campo magnético gerado por um imã de neodimio possa ser representado por $B=k \mu_{0}$, já que para $k=10^{5}$ teríamos, por exemplo, $O(B) \sim 10^{5} .4 \pi \cdot 10^{-7} T \sim O(1000) G \sim O(0,1) T$. Com base nessa observação podemos esperar que a força atuante sobre cada magneto devido ao solenóide nas posições central (c) e extremo (e), seção (j) da Fig. 4, possa ser representada por

$$
\begin{aligned}
& \vec{F}_{c}(B)=\frac{1}{2} \frac{B N^{2} I^{2} S}{\left(L^{2}+D^{2}\right)}(-\hat{i}) \\
& \vec{F}_{e}(B)=\frac{1}{8} \frac{B N^{2} I^{2} S}{\left(L^{2}+\frac{D^{2}}{4}\right)}(-\hat{i}),
\end{aligned}
$$

onde $N$ representa o número de espiras na seção, $D$ é o diametro, $L$ o comprimento da seção, $S=\pi \frac{D^{2}}{4}$ é a área de seção tranversal do solenóide e $B$ o 
campo do imã de neodimio. Como um guia adicional para o estabelecimento das funções desconhecidas, $F(B)$ e $f(I, V, v(t))$, nas linhas seguintes descrevemos brevemente a diferença básica observada no comportamento dos motores homopolares, rotacional e linear, que consiste na observação que os motores entram em regime estacionário em intervalos de tempo distintos. Como é possível observar nos vídeos disponíveis na internet ou fazendo o experimento, o motor linear entra em regime permanente em um intervalo de tempo muito curto, enquanto que o motor rotatório homopolar entra em regime estácionário apenas quando este adquire uma velocidade de rotação suficientemente elevada, o que demanda um tempo considerável. Creditamos este efeito ao fato do fluxo de campo magnético produzido pelos imãs nos dois dispositivos não apresentar o mesmo comportamento. A fim de ilustrar esta observação, na Fig. 5 incorporamos este efeito considerando

$$
\begin{aligned}
& F(B) \propto \frac{N^{2} B S}{\left(L^{2}+\frac{D^{2}}{4}\right)} \\
& f(I, V, v(t)) \propto\left(\frac{V_{\text {pilha }}-A_{r, l} v[t]}{R}\right)^{2},
\end{aligned}
$$

onde as funções acima foram empregadas para plotar $v(t)$ para dois extremos distintos, apenas com a finalidade de exemplificar a observação, e portanto assumimos $\left(A_{l}=1\right.$ e $\left.A_{r}=0,01\right) \operatorname{com} B=0,1 T$, $D=2,0 \mathrm{~cm}, N=6, L=7,0 \mathrm{~cm}, g=10 \mathrm{~m} / \mathrm{s}^{2}, R=$ $0,6 \Omega, V=1,6 \mathrm{~V}, \mathrm{M}=2 \mathrm{~m}_{\text {ima }}+m_{\text {pilha }}=0,0433 \mathrm{Kg}$ e $\mu_{d y n}=0,015$.

De fato para o motor rotacional o fluxo de campo magnético é caracterizado por $\left(\frac{\Delta \Phi}{\Delta t}=B r v_{t}(t)=\right.$ $\left.O(0,01) v_{t}(t)\right)$, e nesse caso podemos identificar

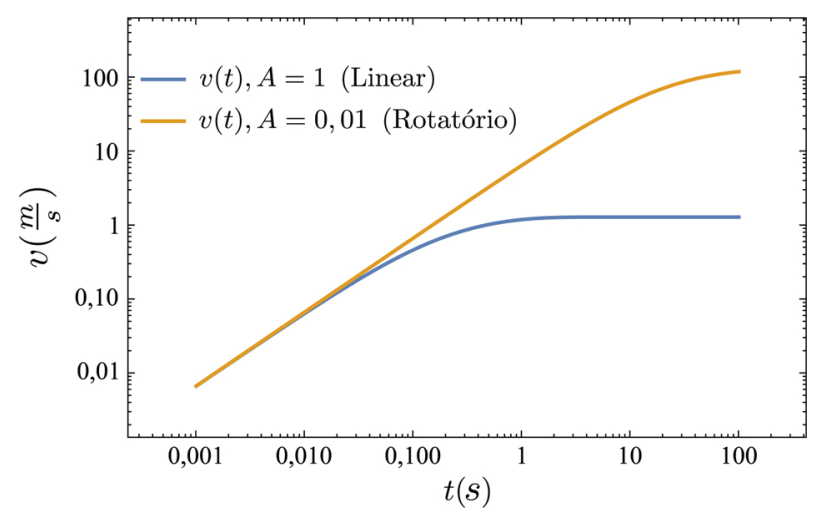

Figura 5: llustraçao da diferença de tempo observado para os dois sistemas para alcançar o regime estacionário
$A_{r}=0,01$. Porém, para o motor linear devemos chamar a atenção neste ponto para uma particularidade da configuração dos magnetos que produzem o movimento. Conforme mostra a Fig. 4(j), devido aos imãs estarem numa configuração de polos magnéticos invertidos, $(S S)$ [centro] e $(N N)$ [extremo], temos um efeito do tipo "lente magnética"para o motor linear de forma que a densidade de linhas de campo dos imãs em (j) é muito menor se comparada a densidade nos extremos. Com o objetivo de caracterizar este efeito, utilizamos um sensor de campo magnético baseado no efeito Hall [10] para determinar experimentalmente a intensidade do campo magnético produzido pelos imãs de neodimio e solenóide. Para coletar e interpretar os dados fornecidos pelo sensor foi utilizada um Arduino Uno, que consiste em uma placa com um micro controlador integrado e plataforma opensource 11. O valor da intensidade do campo magnético gerado pelos imãs, de acordo com a configuração descrita na Fig. 4, está listado na Tabela I.

Considerando a região correspondente a base dos magnetos, o campo medido na superficie dos imãs foi $B=1300 G=0,13 T$, enquanto que o campo registrado a uma distância, $d \approx 2,3 \mathrm{~cm}$, correspondente as posições $(\mathrm{j}+2)$ e $(\mathrm{j}-2)$ na espira foi $B \approx 250 G=0,025 T$. Por outro lado, para a configuração de imãs responsável pelo movimento do motor linear o valor do campo magnetico medido exatamente no mesmo ponto, posição central representado por $(\mathrm{j})$, foi $B=7 G=0,0007 T$, ou seja a configuração de imãs responsável pelo movimento do motor linear leva a uma condição em que o fluxo de linhas de campo dos imãs de neodimio é extremamente reduzido na região central do solenoíde (c) em comparação com o extremo (e), e por este motivo nos referimos a este efeito como "lente magnética", no sentido que as medidas realizadas nos permitiram estimar a diferença de intensidade

Tabela 1: Distribuição da Intensidade do campo magnético obtido para a configuração de imãs representado na Fig. 4. Assumimos como referência a base dos magnetos para inferir a distância (d) em relação a posições $(j),(j+2)$ e $(\mathrm{j}-2)$.

\begin{tabular}{lccc}
\hline$B_{(j)}(\mathrm{T})$ & $B_{(j+2)}(\mathrm{T})$ & $B_{(j-2)}(\mathrm{T})$ & $\mathrm{d}(\mathrm{cm})$ \\
\hline 0,13 & 0,131 & 0,13 & 0,0 \\
0,12 & 0,129 & 0,123 & 1,0 \\
0,015 & 0,034 & 0,031 & 2,0 \\
0,0007 & 0,025 & 0,022 & 2,3 \\
\hline
\end{tabular}


de campo entre estas regiões como sendo da ordem de $\left(\Delta B \approx B_{(j \pm 2)}(G)-B_{(j)}(G) \approx O(250) G\right)$, de forma que podemos inferir a ordem de grandeza para a diferença de fluxo observada entre os dois motores. Finalmente, considerando toda discussão apresentada é possível simular a dinâmica do motor linear considerando

$$
\begin{aligned}
& F(B) \approx \frac{1}{4} \frac{N^{2} B S}{\left(L^{2}+D^{2}\right)} \\
& f(I, V, v(t)) \approx\left(\frac{V_{\text {pilha }}-A_{l} v[t]}{R}\right)^{2},
\end{aligned}
$$

uma vez que na posição central do solenóide, $B \sim$ $0,0007 T$ e $F_{c}(B)<<F_{e}(B)$. Devido a constação que para o motor linear o efeito "lente magnética"leva entre os extremos do magneto $(\Delta B \approx 250 G)$ e a observação que os motores entram em regime estacionário em intervalos de tempo bem distintos $\left(t_{l}<<t_{r}\right)$, por reescala entre o fator de fluxo do caso rotacional, comparado ao linear, esperamos $A_{l} \approx O\left(10^{2}\right) A_{r}$ (vide Fig. 5). Esta foi apenas uma estimativa, baseada na diferença de comportamento observada para as duas versões de motores homopolares e na determinação de $(\Delta B)$ observado para o motor linear. De acordo com o comentário já tecido no início da seção seria preciso a realização de um estudo muito mais detalhado para a descrição da dinâmica e que fugiria do escopo deste traba- lho. Com base no conjunto de variáveis descrito, Eqs.(6),(12) e (13), na Fig.6 apresentamos o comportamento de $v(t)$ em função do atrito dinâmico e do tempo para o modelo teórico proposto para a descrição do motor linear

Como na discussão apresentada após a Fig. 3, a determinação da velocidade terminal irá depender, além de um conhecimento mais detalhado da interação dos magnetos com o campo gerado no solenóide, também da determinação mais precisa do coeficiente de atrito dinâmico dos magnetos com o solenóide. A Fig. 6 nos permite estimar que a velocidade terminal seja limitada superiormente por $(v<0,4 \mathrm{~m} / \mathrm{s})$, resultado este compatível com observado experimentalmente. Na seção seguinte iremos apresentar os resultados obtidos para $v[\mathrm{~cm} / \mathrm{s}]$ e de fato iremos verificar que a estimativa apresentada acima é bem razoável.

\section{Caracterização experimental da veloci- dade do motor linear}

Para determinar experimentalmente a velocidade do sistema (imãs + pilha) utilizamos, para a aquisição de dados, uma câmera digital convencional de um aparelho celular. Então, filmamos o movimento descrito pelo motor após estabelecido o fechamento do circuito elétrico que caracteriza o motor e analisa-

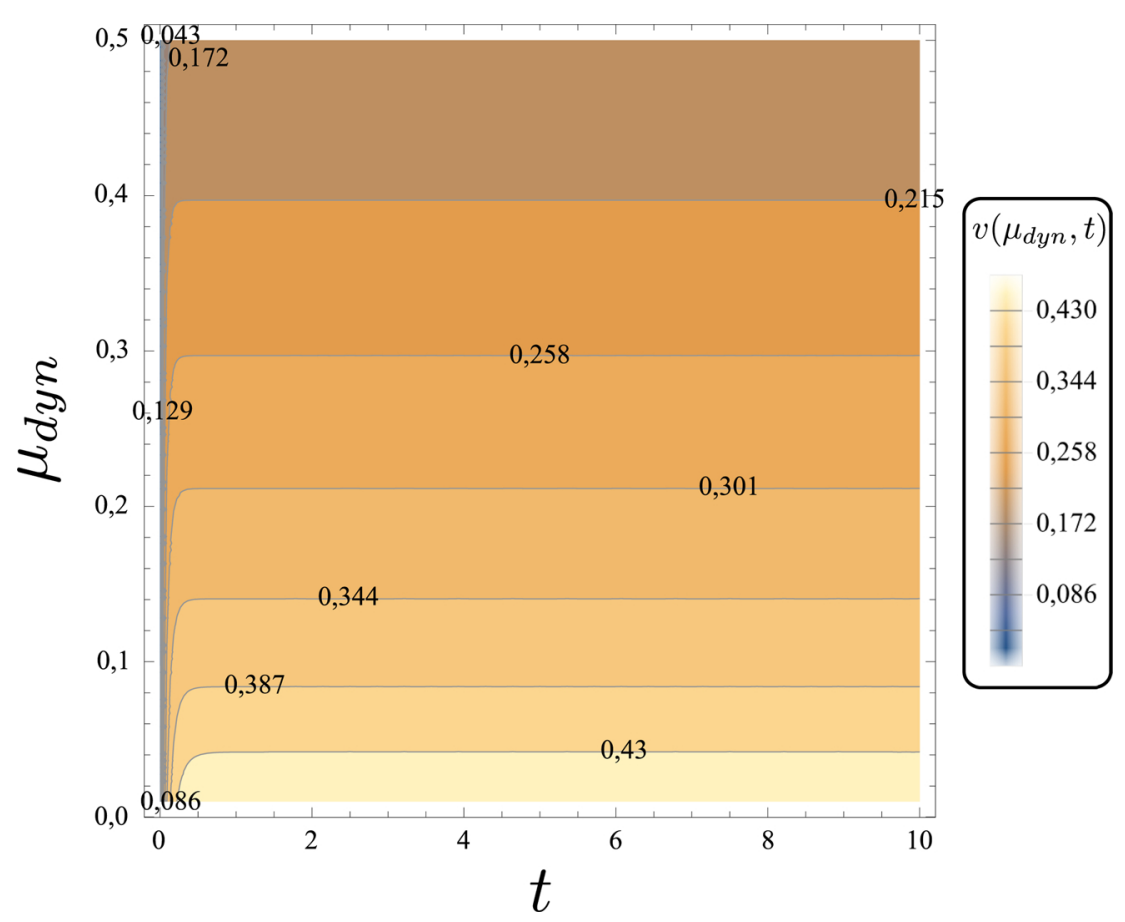

Figura 6: Comportamento de $v(t)$ em função do atrito dinâmico $\left(\mu_{d}\right)$ e do tempo para o motor homopolar linear 
mos o filme com base no programa Tracker [6], que é um software de domínio publico e de fácil utilização, como pode ser visto na Ref. [12]. Na sequência descreveremos com um certo detalhe quais os procedimentos que foram utilizados para aquisição dos dados, primeiramente fixamos a câmera digital sobre o dispositivo como é possível notar na foto exibida na Fig. 2, na sequência, após a câmera ser acionada inserimos na lateral esquerda da espira o conjunto (imãs + pilha), sendo a configuração dos magnetos acoplados a pilha descrito na Fig. 4, após ambos os magnetos entrarem em contato com a espira o movimento do conjunto é registrado pela camera que é responsável em gerar um filme do movimento. Com base no filme gerado a análise dos dados é feita da seguinte forma, considerando o software Tracker 6] é possível converter o filme em uma sequência de quadros onde a posição do conjunto, (imãs + pilha), é registrada a cada quadro por um ponto $P=P(x, t)$ situado no meio da pilha, este ponto é escolhido dentro do ambiente do software sendo reinserido a cada quadro manualmente na posição determinada, uma vez que a pilha está em movimento o ponto central registrado nos quadros posteriores não coincide perfeitamente com a posição registrada no primeiro quadro o que gera, então, a flutuação dos pontos experimentais obtidos, como pode ser verificado na Fig. 7. A partir do número de quadros gerados no intervalo de tempo de duração do filme, o programa produz uma tabela de dados contendo a posição $x_{i}$ para cada instânte $t_{i}$ atribuídos a um dado quadro $(i)$, que então caracteriza a posição do elemento móvel do motor.

A Fig.7 exibe o gráfico gerado a partir do filme obtido. Os resultados experimentais estão descritos por pontos em azul, a linha vermelha representa um ajuste dos dados da forma $x(t)=x_{0}+v_{0} t$, sendo o ajuste empregado para este conjunto de dados $x(t)=0,3737+19,89 t$ que implica em uma velocidade terminal média $\bar{v}=19,89 \mathrm{~cm} / \mathrm{s}$, resultado que está de acordo com a estimativa descrita pela Fig.6, frente as simplificações assumidas.

\section{Ilustrando o funcionamento de um ace- lerador de partículas: Uma aplicação para o motor linear didático}

Aceleradores de partículas podem ser classificados como equipamentos que fornecem energia a feixes de partículas subatômicas eletricamente carregadas.

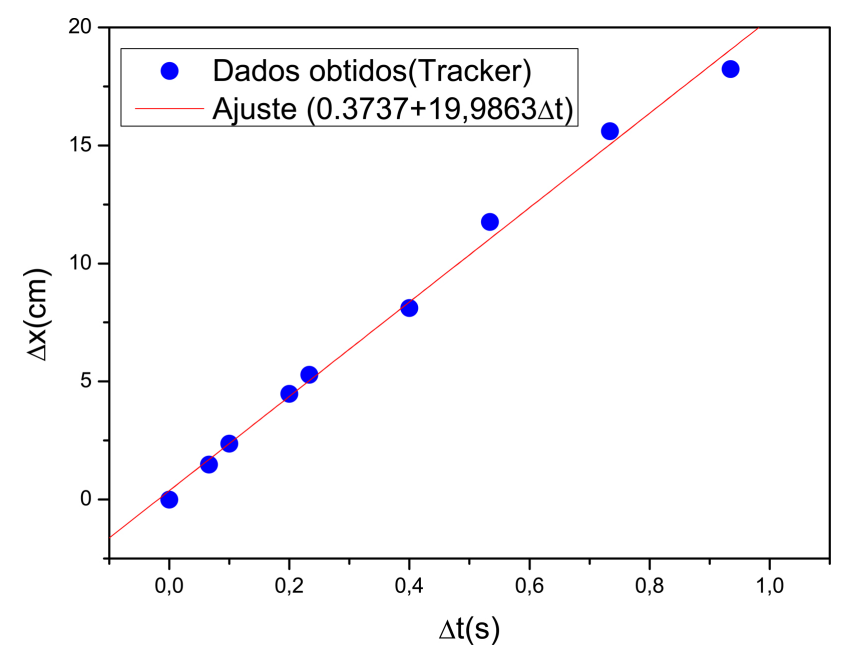

Figura 7: Resultados obtidos para o experimento realizado

Todos os aceleradores de partículas possibilitam a concentração de grande energia em um pequeno volume, e em posições arbitradas e controladas de forma precisa. O exemplo mais elementar de um acelerador de partículas consiste de um tubo da televisão, que nada mais é que um tubo de raios catódicos. Neste caso temos um feixe de elétrons gerado por emissão termo-iônica, que consiste da emissão de elétrons por um filamento aquecido sujeito a uma elevada tensão que proporciona a ejeção destes elétrons, na parte mais fina do tubo estes elétrons são acelerados e focalizados por campos elétricos até atingirem a tela. Podemos separar os aceleradores de partículas em duas classes, Aceleradores Lineares, que iremos ilustrar o funcionamento considerando a dinâmica apresentada pelo motor linear homopolar, e Aceleradores cíclicos. A diferença básica entre os tipos de aceleradores reside no fato que, aceleradores lineares(LINACs) fazem a partícula seguir uma trajetória reta e a energia final obtida pela partícula é proporcional à soma das diferenças de potencial geradas a partir dos mecanismos de aceleração dispostos ao longo da trajetória, enquanto que os aceleradores circulares são elaborados a fim de promover uma trajetória curvada das partículas pela ação dos campos magnéticos. Aceleradores circulares forçam a partícula a passar diversas vezes pelos sistemas de aceleração e a energia final das partículas depende da amplitude da diferença de potencial aplicada, e do número de voltas que estas executam. Uma boa apresentação do princípio de funcionamento de um acelerador linear está postado no site [13] e na Ref. 14, de forma 
geral um acelerador linear é constituído por um tubo muito longo dividido em porções de comprimento variável, conforme representado na Fig. 8.

As seções alternadas do tubo(azul,vermelha) estão conectadas entre si, e uma diferença de potencial periódica do tipo $V(t) \sim V_{0} \sin (\omega t)$ é aplicada nas seções. A figura acima pode ser vista como um "instantâneo"de aplicação de um potencial periódico ao conjunto, por exemplo no instânte capturado pela figura, a partícula adquire energia cinética as custas da energia potencial do campo elétrico estabelecido passando do tubo(0), após um período, chegando ao final do tubo (1) percorrendo a distância $L_{1}$. Sendo o potencial periódico, para que a particula seja acelerada é necessário que seu movimento esteja em fase com o potencial, ou seja após um segundo período de tempo a distância percorrida pela partícula , que adquiriu velocidade $v_{0-1}$ ao transitar na primeira seção do acelerador, deve ser tal que o tempo gasto para percorre-la corresponda ao mesmo período de tempo e, por isso, o comprimento da segunda seção do acelerador deverá obedecer $L_{2}=L_{1} \sqrt{2}$, ou seja $L_{2}>L_{1}$, para que a particula ganhe uma grande quantidade de energia é necessário que o tamanho do acelerador seja proporcionalmente grande, já que para um acelerador composto por $(N-1)$ seções o seu tamanho seria definido por

$$
L_{N}=L_{1} \sqrt{N}
$$

e a energia adquirida sendo $E_{N}=N E_{1}$. A partir dessa observação é possível compreender por exemplo a diferença entre a energia gerada para um feixe de prótons no acelerador projetado por Luis Alvarez em 1946, $\left(L=875 m, E=8 \times 10^{-4} \mathrm{GeV}\right)$, com o SLCStanford em 1987 ( $L=3,2 \mathrm{Km}, \mathrm{E} \sim 50 \mathrm{GeV}) 13$. Finalmente, com base na discussão apresentada podemos agora ilustrar como o motor linear descrito nas seções anteriores pode ser empregado para exemplificar o funcionamento de um acelerador linear de uma forma bastante didática e ilustrativa. Como vimos a partir da Fig. 6, o movimento adquirido pelo conjunto (imãs + pilha), é uma consequência da força magnética resultante sobre os imãs devido ao surgimento de uma corrente na espira. A partir da comparação com o mecanismo de funcionamento de um acelerador linear o conjunto (imãs + pilha) faria o papel da partícula a ser acelerada, no caso do motor linear o ganho de energia cinética do conjunto é uma consequência da variação da densidade de energia magnética apresentada pelo sistema após estabelecido a corrente responsável pelo funcionamento do motor, que faria o mesmo papel da variação da densidade de energia potencial elétrica sentida pela partícula no LINAC. No caso do acelerador linear o ganho de energia cinética da partícula é consequência da inserção de seções com diferentes comprimentos, este mecanismo pode ser ilustrado com o motor didático acrescentando seções de espiras com um aumento progressivo do número de espiras por comprimento de seção, sendo o ganho máximo limitado pelas perdas de energia do conjunto. A fim de ilustrar este efeito na figura 9 apresentamos a velocidade do conjunto para duas situações distintas, espira $\mathrm{A}(\mathrm{L}=20 \mathrm{~cm}, \mathrm{~d}=2$ espiras $/ \mathrm{cm})$ e espira $B(L=50 \mathrm{~cm}, \mathrm{~d}=4$ espiras $/ \mathrm{cm})$, sendo que nesta figura levamos em conta a perda de velocidade devido a dissipação de energia por $\operatorname{ciclo}(\mathrm{N})$.

Assim, para um conjunto contendo $(n)$ seções de espiras conectadas, partindo por exemplo de uma equivalente a descrita na Fig. 2 onde $v_{1} \sim 0,2 \mathrm{~m} / \mathrm{s}$, seria possível com o auxílio da discussão apresentada na seção 3 , por exemplo, efetuar uma estimativa para o tamanho(projeto de acelerador) para a energia final estabelecida para a máquina. Um ponto que deve ser salientado é que a geometria considerada para projeto de acelerador didático, inserção de seções de espiras cilíndricas com um aumento progressivo do número de espiras por comprimento de seção, terá limitações devido ao aumento da resistência ôhmica e indutância do circuito. Entretanto, pelos menos para um número não muito grande de seções de espiras o efeito de aceleração sobre conjunto (imãs + pilha) pode ser observado,

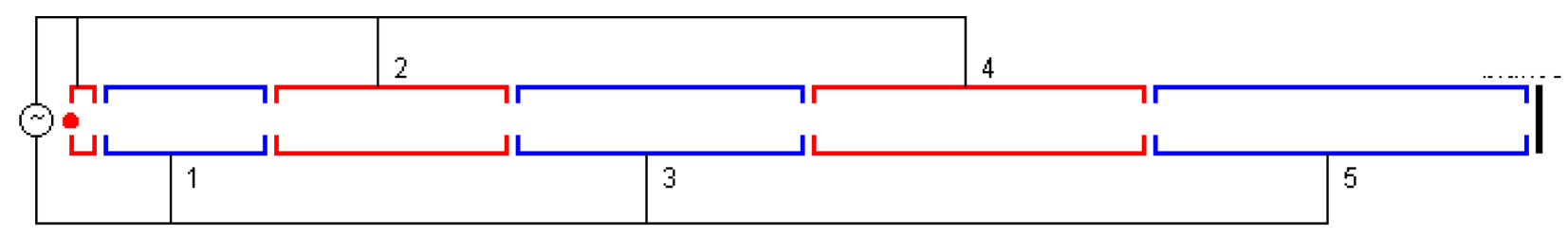

Figura 8: Representação simplificada da estrutura de um acelerador linear de partículas 
por exemplo assumindo os dados que apresentamos na Fig. 9 um acelerador composto pela espiras (A) e (B), que teria o tamanho $(\mathrm{L} \sim 70 \mathrm{~cm})$, ilustraria este efeito uma vez que para um ciclo a velocidade do conjunto, $(\sim 22 \mathrm{~cm} / \mathrm{s})$ espira $(\mathrm{A})$, seria elevado para $(\sim 37 \mathrm{~cm} / \mathrm{s})$ espira (B). Talvez exista alguma outra geometria, não cilíndrica para o formato de espira, que apresente melhores ganhos ficando esta sugestão dirigida ao leitor como uma proposta de projeto de acelerador. Em relação aos resultados apresentados nesta figura devemos notar que a queda da ddp e, portanto, da velocidade deve-se ao aquecimento da pilha, gerado a cada ciclo. O processo químico responsável pela carga da bateria continua a agir, de forma que se aguardarmos um tempo suficiente para a que pilha entre em equilíbrio térmico com o ambiente, $\sim 3 \mathrm{~min}$, a queda na velocidade apresentada na última figura será menor.

\section{Conclusões}

Neste trabalho descrevemos a dinâmica de um motor homopolar linear como um análogo translacional de um motor elétrico homopolar rotacional(usual), como o "roller"homopolar descrito na Ref. [1]. Como uma aplicação ilustramos o princípio de funcionamento de um acelerador de partículas linear. Considerando a distribuição dos assuntos que apresentamos no texto, podemos separar as contribuições do trabalho em três linhas. No tocante ao ensino de Física, as seções 2 e 3 foram dedicadas a apresentação, elaboração e descrição do princípio de funcionamento do motor linear proposto, como vi-

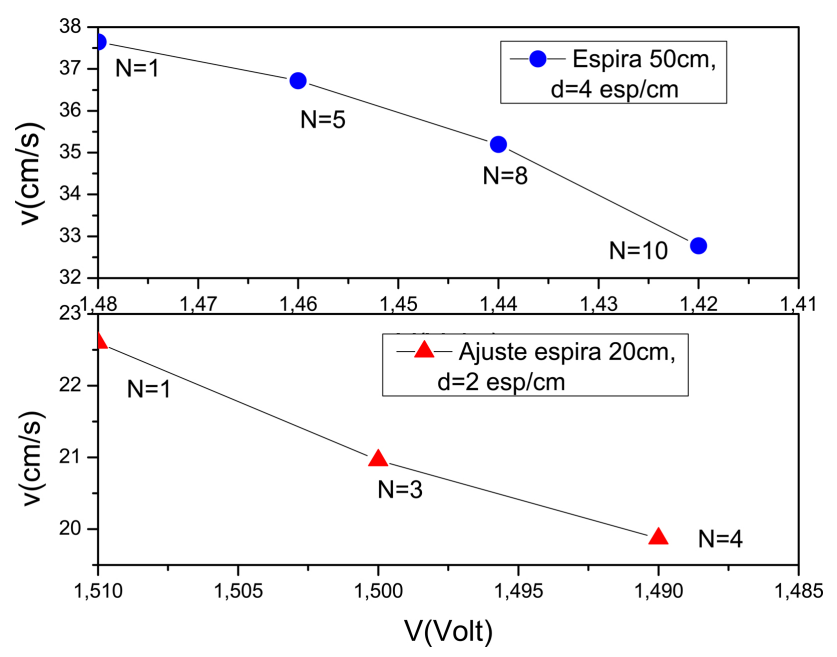

Figura 9: Velocidade obtida para duas espiras (A) e (B) levando em conta a perda por ciclo mos a espira circular (solenóide) é formado por fio elétrico desencapado e que faz o papel de estator de acordo com a definição empregada para um motor linear, uma pilha alcalina AAA é acoplada a dois imãs de neodímio, como os imãs são revestidos por um material condutor ao entrarem em contato com o interior do solenóide estabelecem o fechamento do circuito elétrico. A elaboração de um modelo preciso para a caracterização da dinâmica do motor linear homopolar é uma tarefa complexa, entretanto, considerando uma analogia com o equivalente para o movimento de rotação estabecelecemos os principais conceitos necessários para tal descrição, uma vez que não encontramos na literatura nenhum trabalho orientado a tal descrição. Na seção 4 apresentamos um conjunto de dados, o qual está descrito na Fig. 7, onde determinamos a velocidade do conjunto (imãs + pilha) empregando o software "Tracker" [6].

Desta forma, podemos destacar como nossa principal contribuição a segunda linha, que consiste na apresentação de ferramentas de baixo custo que possam ser empregadas na aquisição e análise dos resultados obtidos. Especificamente, apresentamos a utilização do software "Tracker" [6], do sensor de efeito Hall e o emprego da plataforma arduino, que como salientamos, podem ser facilmente implementados em muitos laboratórios de ensino. Por fim, em relação a aplicação de conhecimentos elementares a descrição de tópicos mais avançados podemos destacar a última seção, na qual exemplificamos o funcionamento de um acelerador linear considerando o motor linear desenvolvido nas seções iniciais. Como vimos a variação da quantidade de movimento adquirido pelo sistema (imãs + pilha) é uma consequência da força magnética resultante sobre os imãs devido ao surgimento de uma corrente na espira. Fazendo uma analogia com o funcionamento de um acelerador linear o conjunto (imãs + pilha) faria o papel da partícula acelerada. No caso do motor linear o ganho de energia cinética do conjunto é uma consequência da variação da densidade de energia magnetica apresentada pelo sistema após estabelecido a corrente responsável pelo funcionamento do motor. No caso do acelerador linear o ganho de energia cinética da particula é devido a inserção de seções com diferentes comprimentos, este mecanismo pode ser ilustrado com o motor didático acrecentando seções de espiras com um aumento progressivo no número espiras por comprimento de 
seção, claro levando em conta a limitação devido às perdas de energia devido a dissipação.

\section{Agradecimentos}

O autor A. Doff agradece ao Conselho Nacional de Desenvolvimento Científico e Tecnológico ( $\mathrm{CNPq})$ pelo apoio financeiro.

\section{Referências}

[1] Seán M. Stewart, Revista Brasileira de Ensino de Física 11, 275 (2007).

[2] https://www youtube.com/watch?v= 25XRekx4WLA; http://www.videoman.gr/pt/ 74359

[3] Christopher J. Chiaverina, The Physics Teacher 42, 553 (2004).

[4] Davi Featonby, Physics Education 41, 292 (2006).

[5] Stephen Irons, The Physics Teacher 53, 186 (2015).

[6] Douglas Brown, Tracker: Free Video Analysis and Modeling Tool for Physics Education, disponível em http://www . cabrillo.edu/ dbrown/tracker.

[7] Hugo Sakai Idagawa, Projeto, Montagem e Caracterização de Motor Linear de Imã Permanente. Trabalho de Graduação II, Unicamp, 2009.

[8] Milton Antonio Zaro, Rosa Leamar Dias Blanco e Carlos Alberto Kern Thomas, Revista Brasileira de Ensino de Física 11, 88 (1989).

[9] Magmattec: Solutions in Magnetic Materials, disponível em http://www.magmattec.com/ products.html.

[10] Continuous-Time Ratiometric Linear Hall Effect Sensor ICs (Alegro MicroSystems), disponível em http://www.allegromicro.com/en/Products/ Magnetic-Linear-And-Angular-PositionSensor-ICs/Linear-Position-Sensor-ICs/ A1301-2.aspx.

[11] Arduino Playground HomePage, disponível em http: //www .arduino.cc.

[12] Adriano Doff, Jalves S. Figueira e Jean C. Gentilini, Revista Brasileira de Ensino de Física 33, 1305 (2011).

[13] Wikipedia contributors, Particle Accelerator, disponível em https://en.wikipedia.org/wiki/ Particle-accelerator.

[14] Rui Alvez Pires e Rui Dilão, Gaz. Phys. 15, 56 (1992). 